\title{
In vitro dissolution and in vivo gamma scintigraphic evaluation of press-coated salbutamol sulfate tablets
}

\author{
WEI LI ${ }^{1}$ \\ CAI-HONG SHI ${ }^{1}$ \\ YI-LING SHENG ${ }^{1}$ \\ PING CUI ${ }^{2}$ \\ YU-QING ZHAO \\ XIANG-RONG ZHANG ${ }^{1 *}$ \\ 1 Shenyang Pharmaceutical University \\ Shenyang 110016, Liaoning Province \\ China \\ 2 The First Hospital of China Medical \\ University, Shenyang 110001, Liaoning \\ Province, China
}

Accepted July 3, 2013

\begin{abstract}
The aim of this study was to investigate the in vitro and in vivo performance of salbutamol sulfate press-coated tablets for delayed release. The in vitro release behavior of press-coated tablets with the outer layer of PEG 6000/ Eudragit S100 blends (2:1) in pH $1.2\left(0.1 \mathrm{~mol} \mathrm{~L}^{-1} \mathrm{HCl}\right)$ and then $\mathrm{pH} 6.8$ buffer solution was examined. Morphological change of the press-coated tablet during in vitro release was recorded with a digital camera. Release of salbutamol sulfate from press-coated tablets was less than $5 \%$ before $3 \mathrm{~h}$ and was completed after $8 \mathrm{~h}$ in $\mathrm{pH}$ 6.8 phosphate buffer solution. In vivo gamma scintigraphy study carried out on healthy men indicated that the designed system released the drug in lower parts of the GI tract after a lag time of 5 hours. The results showed the capability of the system of achieving delayed release of the drug in both in vitro and in vivo gamma scintigraphy studies.
\end{abstract}

Keywords: salbutamol sulfate, press-coated tablet; PEG 6000-Eudragit S100 blends, dissolution, gamma scintigraphy

To combat the early morning mortality associated with the pathophysiological diseases of myocardial infarction and bronchial asthma, therapeutically active concentrations of the drug are required in the early morning hours just prior to awakening (1). This cannot be achieved with the conventional controlled-release formulations with zero order kinetics. Therefore, the treatment should preferentially involve formulations that release drugs rapidly after a defined delay period (2). Delayed-release systems mimic the circadian rhythm of the disease by releasing the drug at the appropriate time when the dosage forms come in contact with gastrointestinal fluids (3).

Delayed-release formulations generally have a rapidly disintegrating tablet core encompassed by a barrier layer formed by press-coating, polymer coating or a combination of both (4). Compared to polymer coating, the press-coating technique has the ad-

\footnotetext{
*Correspondence; e-mail: zhangxr@vip.sina.com
} 
vantage of being used without solvents and requiring a relatively short manufacturing process. Composition of the barrier layer controls the mechanism of effecting the lag time. Polymer blends could be a solution for creating a new composition, exhibiting the appropriate flexibility for the adjustment of drug release delay $(5,6)$.

Salbutamol sulfate (STS) is one of the most widely used drugs for the treatment of bronchial asthma, chronic bronchitis and emphysema (7). Treating diseases such as asthma, with immediate-release dosage forms may not be effective if the disease is more pronounced early in the morning. There is a need for a specially designed drug delivery system that could maintain STS concentration at the therapeutic level for some time. STS falls under Class I (high solubility-high permeability) drug according to the Biopharmaceutical Classification System and is absorbed throughout the gastrointestinal tract (8, 9).

In our previous study, we investigated the optimized formulation of a press-coated tablet of salbutamol sulfate using polyethylene glycol 6000 (PEG 6000)-Eudragit S100 dispersion blends in the outer shell and evaluated its pharmacokinetic behavior in beagle dogs (10). The objective of the present work was to investigate the morphological change of the press-coated tablet during dissolution in in vitro and in vivo gamma scintigraphic studies carried out on healthy men.

\section{EXPERIMENTAL}

\section{Materials}

Salbutamol sulfate was obtained from YaBang Co. Ltd. (Changzhou, China). Sodium carboxymethyl starch (CMS-Na; DMV, Veghel, The Netherlands), low-substituted hydroxypropyl cellulose (L-HPC, Shin-Etsu Company, Tokyo, Japan), lactose (BoDi Co. Ltd., Tianjin, China), polyvinylpyrrolidone (PVP, Fengli Jingqiu Commerce \& Trade Co. Ltd., Tianjin, China), and magnesium stearate (BoDi Co. Ltd., Tianjin, China) were used for the preparation of core tablets. PEG 6000 (Kermel Chemical Reagents Development Centre, Tianjin, China) and Eudragit S100 (supplied as a gift by Degussa Co. Ltd. Shanghai Branch, China) were used for the outer shell of timed-release press-coated tablets. Technetium-99m-diethylentriamine pentaacetic acid (99mTc-DTPA) was obtained from the Department of Nuclear Medicine of the Shenyang General Hospital of Military Area Command (Shenyang, China). All other chemicals and solvents were of standard pharmaceutical grade.

\section{Preparation of press-coated salbutamol sulfate tablets}

A wet granulation method was applied to prepare granules for the core tablet. STS core tablets were compression-coated with $150 \mathrm{mg}$ PEG 6000-Eudragit S100 polymer blends in a $2: 1 \mathrm{~m} / \mathrm{m}$ ratio prepared by the fusion method. Each core tablet consisted of 9.6 mg STS, $10 \mathrm{mg}$ CMS-Na, $20 \mathrm{mg}$ L-HPC and $20 \mathrm{mg}$ lactose. The mixture was wetted with $3 \%(\mathrm{~m} / \mathrm{V})$ polyvinylpyrrolidone ethanol solution as binder. The wetted mass was forced through a $920 \mu \mathrm{m}$ screen. The granules were dried at $50{ }^{\circ} \mathrm{C}$ and then sized by passing 
through a $1060 \mu \mathrm{m}$ screen. Next, $0.5 \%$ magnesium stearate, as lubricant based on total mass, was added to the granules and mixed for 5 minutes so that the particle surface was evenly coated by the lubricant. The granules were compressed at a compression force of $4 \mathrm{~kg} \mathrm{~mm}^{-2}$ using a single punch tablet machine equipped with a concave punch $6 \mathrm{~mm}$ in diameter (Jingcheng Machine Manufacture Co. Ltd., Shandong, China). The coating layer was made of PEG 6000-Eudragit S100 polymer blends in a 2:1 ( $\mathrm{m} / \mathrm{m})$ ratio. The required amount of polymer mixture was heated in a stainless steel vessel at $95-99{ }^{\circ} \mathrm{C}$, followed by stirring until homogeneous. It was then allowed to cool to room temperature and kept in the refrigerator at $0-4{ }^{\circ} \mathrm{C}$ for two hours. After that, the mass was ground into a very fine powder prior to use and $0.5 \%$ magnesium stearate, as lubricant based on total mass, was added. Press-coated tablets were prepared as follows: one half of the mixed powder was filled into the die, and a core tablet was placed in the center of the polymer. Then, the remaining half was filled in the die, and the entire content was compressed. The coating material was compressed around the core tablet applying a force of $3 \mathrm{~kg}$ $\mathrm{mm}^{-2}$ with $9.0 \mathrm{~mm}$ round concave punches using a single-punch tablet machine.

\section{Preparation of radiolabelled tablets for the scintigraphy study}

The core tablet (average mass $50 \mathrm{mg}$ ) for the in vivo scintigraphic study consisted of

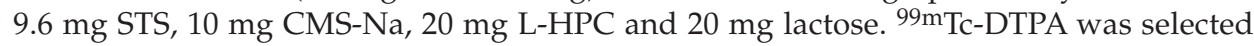
to radio label the tablets because of its short half-life of $6 \mathrm{~h}$ and very low electron emission. Lactose was used as filler and $5 \mathrm{mCi}$ 99m Tc-DTPA was adsorbed on it. Core tablets containing ${ }^{99 \mathrm{~m} T c-D T P A}$ were prepared by radiolabelling DTPA with a sodium pertechnetate solution. Lactose was dissolved in this solution, evaporated to dryness. The radio-labeled core tablets were then press-coated using the preparation method described above.

\section{In vitro drug release studies}

The dissolution study was performed according to the third method (paddle type) described in part II of Pharmacopoeia of China (2010), using a paddle apparatus at 100 rpm. Dissolution media consisted of $100 \mathrm{~mL}$ of $0.1 \mathrm{~mol} \mathrm{~L}^{-1} \mathrm{HCl}$ for the first $2 \mathrm{~h}$ and $\mathrm{pH}$ 6.8 phosphate buffers for the following $7 \mathrm{~h}$ at $37 \pm 0.5^{\circ} \mathrm{C}$. Samples of $5 \mathrm{~mL}$ were withdrawn at specified time intervals and filtered $(0.45 \mu \mathrm{m})$. STS concentrations in sample solutions were analyzed at $276 \mathrm{~nm}$ for STS using a UV9100 spectrophotometer (Rili Analytical Instrument Co. Ltd., Beijing, China). An equivalent volume of temperature-equilibrated dissolution medium was replaced into the dissolution vessel following the removal of each sample. Data represent the mean values of at least six separate experiments.

\section{Photographic study}

At predetermined time intervals $(2,3,4$ and $5 \mathrm{~h})$, press-coated tablets were carefully removed from the dissolution apparatus and images of the press-coated tablets were obtained using a digital camera (Canon A590, Japan). When the press-coated tablets were partly eroded after $6 \mathrm{~h}$, images were taken in the dissolution media. 


\section{In vivo gamma scintigraphic study}

For delayed-release systems, gamma scintigraphy is a well established and useful tool to monitor the dosage form location within the GI tract and assess its in vivo performance (11). Visual tracking of dosage forms is of great importance if the performance of the barrier layer depends upon its location within the GI tract. Two healthy males, 21 and 24 years old, 1.74 and $1.76 \mathrm{~m}$ tall and weighing 60 and $62 \mathrm{~kg}$, respectively, participated in the study as volunteers. They were non-alcoholics, non-smokers, without a history of gastro-intestinal disorders and were not taking any regular medication. The purpose of the study was fully explained and they had given their informed consent in writing. The study was approved by the Independent Ethics Committee of the General Hospital of Shenyang Military Command and was in full compliance with the principles of the Good Clinical Practice guidelines.

After overnight fasting, the volunteers were given breakfast consisting of eggs, bread and milk. Three hours later, they were given $200 \mathrm{~mL}$ of water to which $20 \mathrm{mCi}$ ${ }^{99} \mathrm{~m}_{\mathrm{TcO}}{ }_{4}^{-}$had been added for the purpose of outlining the gastrointestinal tract. Radio-labeled tablets were then administered to volunteers 1 and 2. Gamma images were recorded using an online computer system (Millennium VG hawk-eye, USA) and static 10-s anterior images were acquired at hourly intervals. Gastrointestinal transit of the dosage forms was monitored and their break-up time was assessed.

\section{RESULTS AND DISCUSSION}

\section{In vitro drug release of press-coated STS tablets}

Fig. 1 shows the results of the salbutamol sulfate release from a press-coated tablet. A very small amount of drug was released over the initial period in acidic to weakly acidic medium. However, with an increase of $\mathrm{pH}$, hydration and swelling of the PEG 6000 /Eudragit S100 blends resulted in the rupture of the coating layer, followed by drug release. In this study, $4 \mathrm{~h}$ lag time of release was calculated from the intersection of the linear portion of release with the x-axis representing the time scale. As the drug release behavior shows, the release of STS was less than $5 \%$ before $3 \mathrm{~h}$ and was completed after $8 \mathrm{~h}$ in a $\mathrm{pH} 6.8$ phosphate buffer solution.

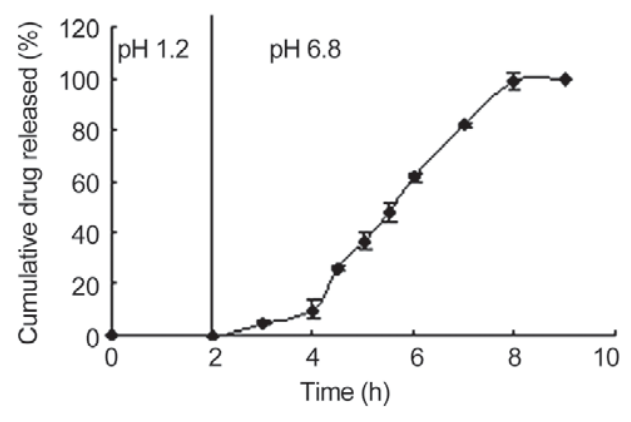

Fig. 1. Dissolution profiles of the press-coated STS table in dissolution media of $0.1 \mathrm{~mol} \mathrm{~L}^{-1}$ $\mathrm{HCl}$ and $\mathrm{pH} 6.8$ phosphate buffers. Each point represents the mean of three determinations. 


\section{Photographic study}

Visual changes of STS tablets press-coated with PEG 6000-Eudragit S100 blends (2:1, $\mathrm{m} / \mathrm{m}), 150 \mathrm{mg}$, are shown in Fig. 2. Very clear correlation between rupturing time and drug release onset was found by visual observation of the tablet during its release. The photographs were taken at recorded time intervals in order to reveal the changes of the outer layer during the drug release. It is clear that the tablet remained intact and smooth in $0.1 \mathrm{~mol} \mathrm{~L}^{-1} \mathrm{HCl}$ for the first $2 \mathrm{~h}$ (Fig. 2a) because the dissolution medium did not penetrate into the PEG 6000-Eudragit S100 coating. Thus, after the contact of the outer layer with the $\mathrm{pH} 6.8$ phosphate buffer solution, the surface of the tablet appeared rough due to hydration and swelling after $3 \mathrm{~h}$ (Fig. 2b) and drug release increased. The first small crack on the surface of the outer layer was observed after $4 \mathrm{~h}$ (Fig. 2c). At the same time, the released amount of the drug started to increase. After $\mathrm{pH} 6.8$ phosphate buffer had penetrated into the outer layer, the crack spread in the outer layer after $5 \mathrm{~h}$ and approximately $40 \%$ of the drug was released (Fig. 2d). The outer layer was disintegrated and internal STS tablet was visible. After $6 \mathrm{~h}$, in the $\mathrm{pH} 6.8$ phosphate buffer solution (Fig. 2e), STS was partly exposed to the dissolution medium leading to $60 \%$ drug release. This enabled subsequent complete release of the drug.
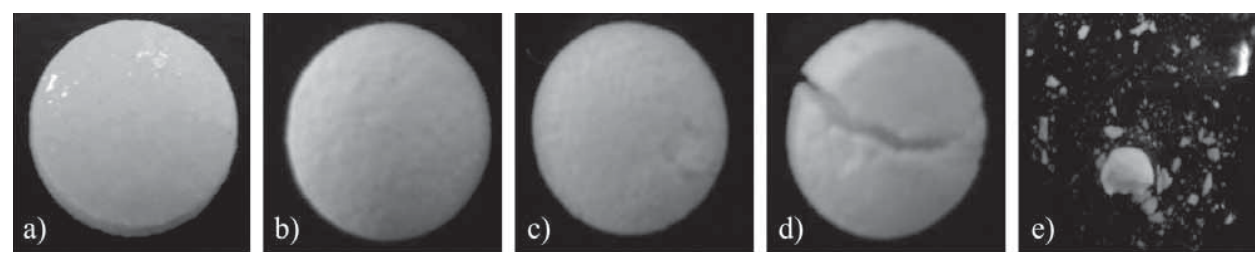

Fig. 2. Morphological changes of the press-coated tablet at various time intervals during dissolution testing: a) $2 \mathrm{~h}$, b) $3 \mathrm{~h}, \mathrm{c}) 4 \mathrm{~h}, \mathrm{~d}) 5 \mathrm{~h}$ and e) $6 \mathrm{~h}$.

\section{In vivo gamma scintigraphic studies}

Press-coated tablets of STS were administered to two men to examine acid resistance and delayed-release performance of STS in the gastrointestinal tract. Gamma scintigraphy of the optimized formulation was performed in healthy human volunteers in order to assess the in vivo drug release behavior.

Gamma scintigraphy is currently considered to be the non-invasive technique of choice for determining the in vivo performance of dosage forms (4). Gamma scintigraphic images of volunteers 1 and 2 are shown in Fig. 3a,b. The discrete bright spots recorded in sequential images indicated that the tracer incorporated in the active core was not released during the first five hours after administration. Acid resistance of the outer barrier layer of the press-coated tablets protected the drug in the active core from the acid environment during the gastric residence period. After 5 hours of administration, the tablets began to release the tracer, suggesting that the press-coated layer began to play its delayed-release function. The tablets were disintegrated after $7 \mathrm{~h}$, as evident from the distribution of the tracer in the different parts of the colon shown in Fig. 3. 
W. Li et al.: In vitro dissolution and in vivo gamma scintigraphic evaluation of press-coated salbutamol sulfate tablets, Acta Pharm. 63 (2013) 545-551.
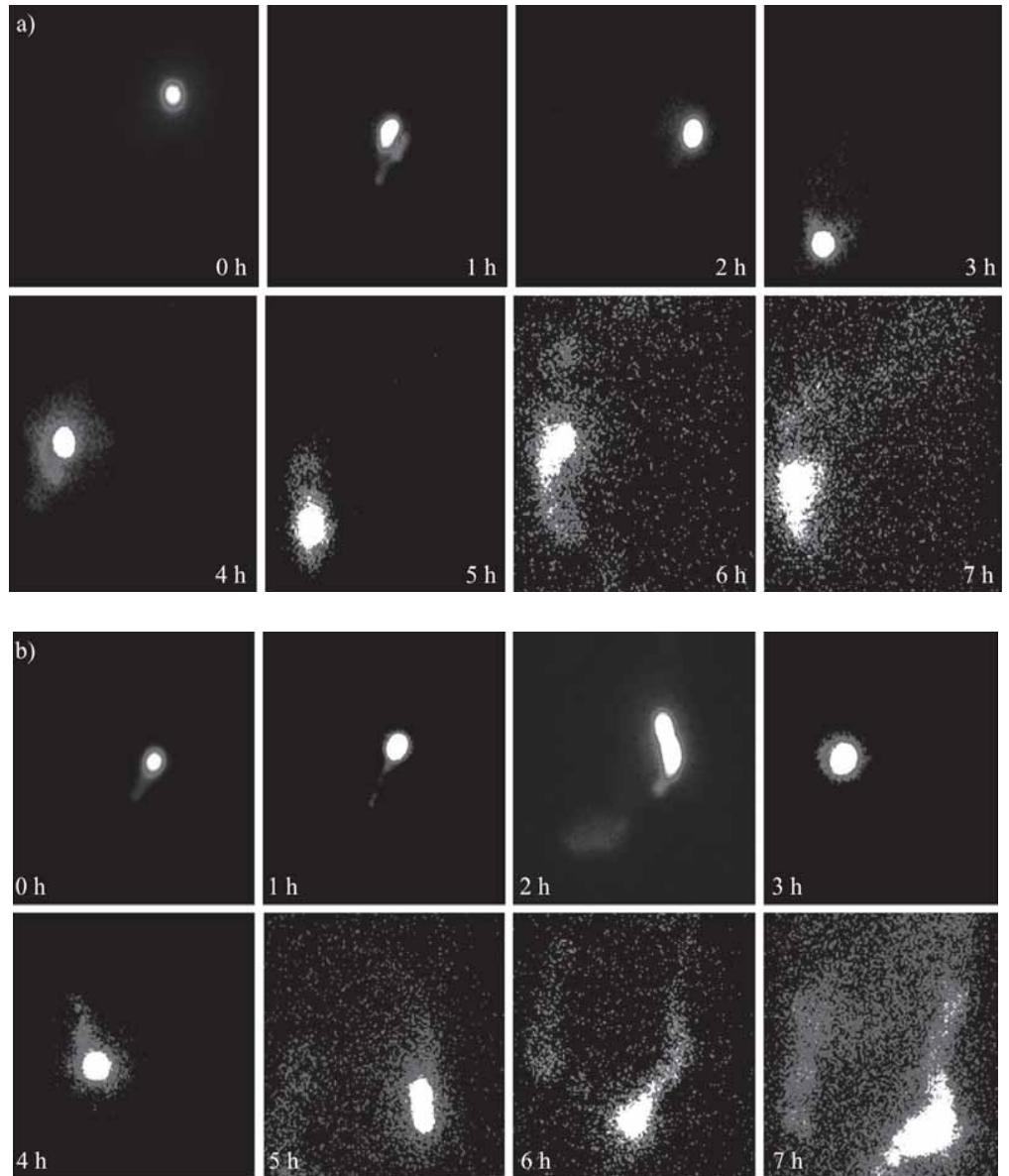

Fig. 3. Gamma scintigraphic images of the press-coated tablet at different time intervals: a) volunteer 1 and $b$ ) volunteer 2 .

\section{CONCLUSIONS}

Delayed release tablets with salbutamol sulfate as a core and homogenous blend of PEG 6000 and Eudragit S100 as outer barrier layer were developed. The press-coated salbutamol sulfate tablet can produce a successful drug release for a programmable period of time based on release in vitro as well as in vivo gamma scintigraphic study.

Acknowledgement. - The authors appreciate financial support from the Natural Science Foundation of Liaoning (Grant No. 20111135) and the national Natural Science Funds of China (Grant No. 81302662). 


\section{REFERENCES}

1. N. Nidni, Chronotherapeutics - a chronopharmaceutical approach to drug delivery in the treatment of asthma, J. Control. Release 163 (2012) 353-360; DOI: 10.1016/j.jconrel.2012.09.012.

2. K. P. Pagar and P. R. Vavia, Felodipine $\beta$-cyclodextrin complex as an active core for time delayed chronotherapeutic treatment of hypertension, Acta Pharm. 62 (2012) 395-410; DOI: 10.2478/ v10007-012-0023-0.

3. L. ShanYang and K. Yoshiaki, Current status and approaches to developing press-coated chronodelivery drug systems, J. Control. Release 157 (2012) 331-353; DOI: 10.1016/j.jconrel.2011.09. 065 .

4. M. Ghimire, F. J. McInnes, D. G. Watson, A. B. Mullen and H. N. E. Stevens, In-vitro/in-vivo correlation of pulsatile drug release from press-coated tablet formulations: A pharmacoscintigraphic study in the beagle dog, Eur. J. Pharm. Biopharm. 67 (2007) 515-523.

5. G. P. Andrews, D. S. Jones, O. A. Diak, C. P. McCoy, A. B. Watts and J. W. McGinity, The manufacture and characterisation of hot-melt extruded enteric tablets, Eur. J. Pharm. Biopharm. 69 (2008) 264-273; DOI: 10.1016/j.ejpb.2007.11.001.

6. T. Yoshihiro, O. Hiroyuki and O. Makoto, Non-invasive and rapid analysis for observation of internal structure of press-coated tablet using X-ray computed tomography, Drug. Dev. Ind. Pharm. 35 (2009) 678-682; DOI: 10.1080/03639040802587807.

7. H. W. Kelly and S. Murphy, Beta-adrenergic agonists for acute, severe asthma, Ann. Pharmacother. 26 (1992) 81-91.

8. C. Xiaoyu, H. Wenhua, W. Blenda Chi, Y. Linlin, W. Yuen Fan, X. Min and Y. Zhijun, Liposomes prolong the therapeutic effect of anti-asthmatic medication via pulmonary delivery, Int. J. Nanomedicine 7 (2012) 1139-1148; DOI: 10.2147/IJN.S28011.

9. A. Paratchikody, V. V. Prasanth, S. T. Mathew and B. A. Kumar, Development and characterization of mucoadhesive patches of salbutamol sulfate for unidirectional buccal drug delivery, Acta Pharm. 61 (2011) 157-170; DOI: 10.2478/v10007-011-0011-9.

10. W. Jing, C. Ping, L. Qiuhua, L. Sanming, W. Ling and Z. Xiangrong, Development and evaluation of press-coated salbutamol sulfate tablets for delayed-release dosage forms, J. Drug Del. Sci. Tech. 22 (2012) 335-334.

11. H. N. Stevens, C. G. Wilson, P. G. Welling, M. Bakhshaee, J. S. Binns, A. C. Perkins, M. Frier, E. P. Blackshaw, M. W. Frame, D. J. Nichols, M. J. Humphrey and S. R. Wicks, Evaluation of Pulsincap to provide regional delivery of dofetilide to the human GI tract, Int. J. Pharm. 236 (2002) 27-34. 